\title{
Hallazgos Histopatológicos del Tejido Pericoronario de Terceros Molares Incluidos Sanos
}

\author{
Histopathological Findings of Pericoronary Tissue in Healthy Third Molars
}

Parra V. \& Ibaceta G.

PARRA, V. \& IBACETA, G. Hallazgos histopatológicos del tejido pericoronario de terceros molares incluídos sanos. Int. J. Odontostomat., 13(3):332-337, 2019.

RESUMEN: Este estudio se realizó para evaluar la presentación de cambios histopatológicos tempranos en sacos pericoronarios de terceros molares incluidos sanos. Sesenta y un tejidos pericoronarios de terceros molares incluidos, clínicamente asintomáticos y sin evidencia radiográfica de anomalía del saco pericoronario, se extrajeron por diferentes razones. Las muestras se fijaron y procesaron de forma rutinaria y se tiñeron con hematoxilina-eosina antes de la evaluación. Se estandarizaron los diagnósticos concluyendo que toda muestra que presente metaplasia escamosa del epitelio reducido del órgano del esmalte (EEE) se considerará cambio quístico temprano. De las muestras presentadas, el $28 \%$ mostró metaplasia escamosa sugerente de cambio quístico temprano. Esta variación histológica del tejido pericoronario fue significativamente mayor en los pacientes menores de 20 años de edad, sexo femenino, terceros molares mandibulares, en inclusión ósea y en pacientes que no informaron sintomatología local previa $(P=0,05)$. Estos hallazgos sugieren que la ausencia de semiología clínica y radiográfica en terceros molares incluidos no necesariamente indica ausencia de alteraciones en el tejido pericoronario de los mismos.

PALABRAS CLAVE: tejido pericoronario, tercer molar, histopatología.

\section{INTRODUCCIÓN}

Los terceros molares incluidos son el motivo de consulta de mayor demanda de los Cirujanos Bucales y Máxilo-faciales.

Así, la decisión de extraer un diente es simple cuando los signos y síntomas de la patología están presentes, pero se hace más complejo cuando el paciente está asintomático. Frente a ello, diversos autores han demostrado que terceros molares asintomáticos son potenciales causantes de enfermedad periodontal de piezas vecinas (Moss et al., 2006), alto potencial riesgo de generación de caries (Divaris et al., 2012) y posibilidad de desplazamiento en el tiempo (Phillips et al., 2007), todos factores que podrían considerar la realización de una extracción "profiláctica", idealmente a edad temprana (menos de 25 años) por la bajas complicaciones que el procedimiento conlleva.

Dentro de estas posibles indicaciones, en la literatura se ha mencionado sobre el potencial pa- tológico de los tejidos pericoronario de los terceros molares incluidos, especialmente en la posible generación de quistes, sin embargo, esto aún no es claro debido a que rutinariamente solo se efectúa un análisis histopatológico en aquellos casos en que se sospecha de alguna patología evidenciada mediante el examen clínico y radiológico.

El diagnóstico de las alteraciones patológicas de los maxilares se determina a través un análisis histológico-radiográfico. Así, para poder indicar la extracción de un tercer molar incluido con cierta certeza de que su tejido pericoronario corresponde a un tejido patológico, debemos contar con una imagen radiográfica fuera de los rangos normales. Pero, ¿Qué hacemos cuando tenemos un paciente clínicamente asintomático y con sacos pericoronarios radiográficamente dentro de los rangos normales? Generalmente en esta situación no suele pensarse en alguna variación patológica, por lo que el diagnóstico solo se efectuaría en etapas 
posteriores o cuando la lesión ha alcanzado un tamaño considerable. Frente a esta problemática, la realización de este estudio permitirá evaluar la presentación de cambios histopatológicos tempranos, fundamentalmente quísticos, en sacos pericoronarios sin evidencia clínica ni radiográfica de alteración patológica, con el fin de conocer el potencial daño al que podrían quedar expuestos aquellos pacientes cuyo profesional tratante o ellos mismos deciden no realizar la extracción por indicación profiláctica.

\section{MATERIAL Y MÉTODO}

Se realizó un estudio descriptivo-transversal para determinar la prevalencia de cambios patológicos en sacos pericoronarios asociados a terceros molares incluidos sanos mediante un análisis histopatológico. El estudio consta de una muestra total de 61 biopsias de sacos pericoronarios de terceros molares sanos en pacientes diferentes. Las muestras fueron obtenidas en el pabellón de la Facultad de Odontología UNAB Santiago, previo consentimiento informado firmado por cada paciente, durante los meses de Mayo hasta Septiembre del año 2018. Las cirugías de terceros molares incluidos sanos, fueron programadas por diferentes indicaciones de extracción. cientes:

Se incluyeron en el estudio los siguientes pa-

1. Pacientes de edad entre 12 y 40 años.

2. Diente asintomático al momento de la toma de biopsia del saco pericoronario en pabellón.

3. Diente incluido, superior o inferior, con integridad intraoperatoria de saco pericoronario suficiente para realización de biopsia.

Se excluyeron del estudio los siguientes pacientes:

1. Pacientes con enfermedades sistémicas descompensadas que no puedan someterse a la cirugía en pabellón.

2. Pacientes con patología relevante que pueda afectar la condición del tercer molar retenido (Enfermedades y/o síndromes que afecten la odontogénesis).

3. Evidencia radiográfica de anomalía del saco pericoronario o de los tejidos peridentarios.
Las muestras obtenidas fueron fijadas (Formalina al $10 \%$ ), y enviadas a estudio histológico al laboratorio de histopatología UNAB, Viña del Mar, donde fueron preparadas, analizadas y diagnosticadas mediante una técnica histológica de rutina utilizando tinción de Hematoxilina-eosina (HE). Se realizó su evaluación microscópicamente a través de microscopía de luz y $400(40 \times 10)$ magnificación (Figs. 1, 2 y 3).

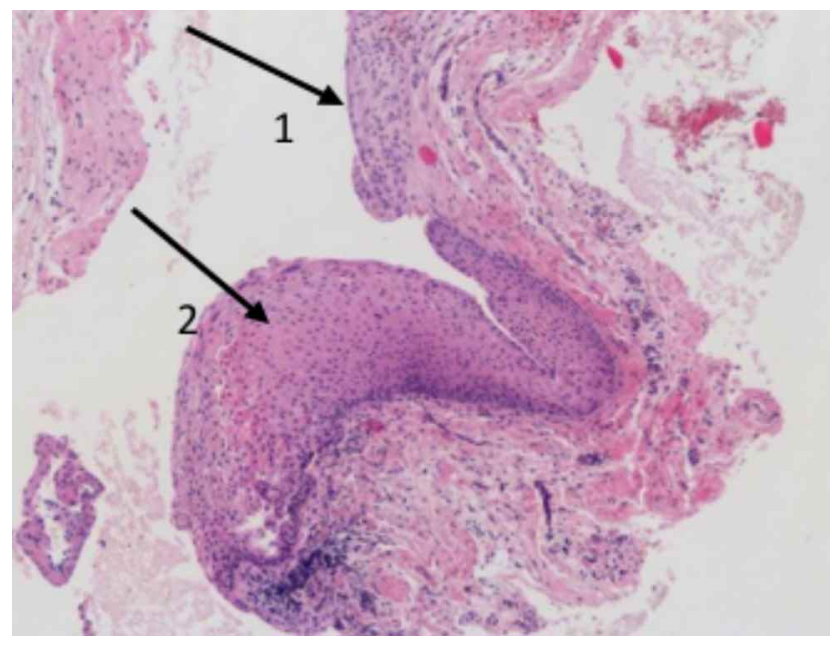

Fig. 1. Porción epitelial con metaplasia escamosa del epitelio reducido del órgano del esmalte (flecha 1) asociada un tejido conectivo denso, presencia de restos epiteliales odontogénicos e infiltrado inflamatorio crónico. En la porción restante se puede apreciar epitelio reducido del órgano del esmalte (flecha 2) (Tinción H-E 10x).

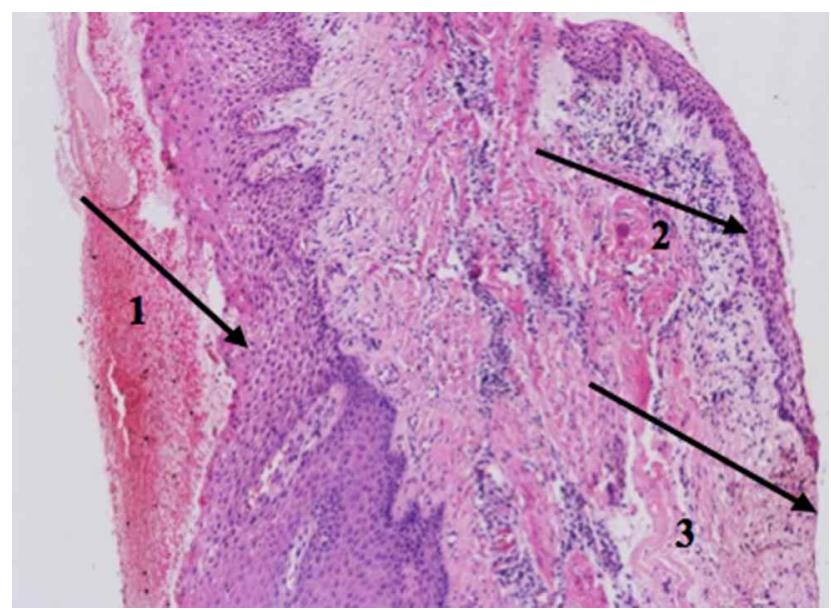

Fig. 2. Pericoronaritis crónica. Se aprecia epitelio de mucosa oral (flecha 1), una interfase de conectivo con infiltrado inflamatorio crónico intenso y el revestimiento epitelial del saco pericoronario donde en una porción presenta metaplasia escamosa del epitelio reducido del órgano del esmalte (flecha 2) y en la otra epitelio reducido (flecha 3) (Tinción H-E 40x). 

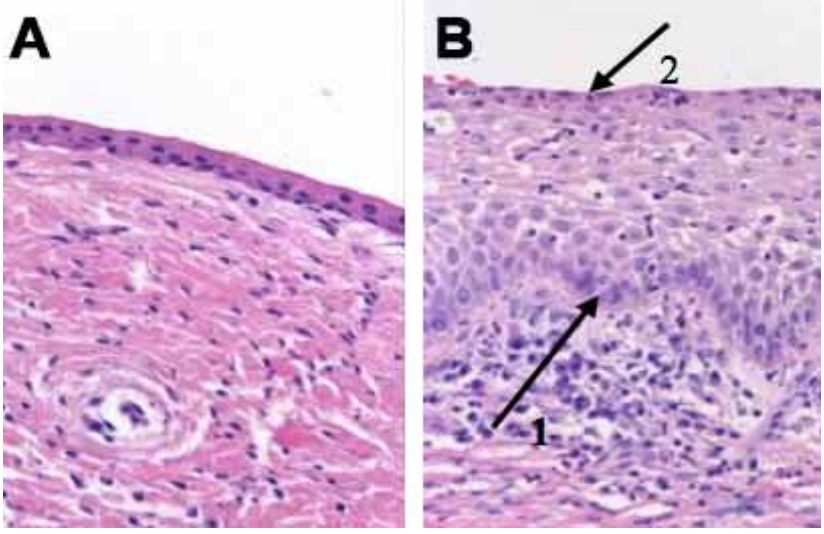

Fig. 3. A: Saco pericoronario normal. Epitelio reducido del órgano del esmalte compuestos por dos capas de células, una cuboidal y otra plana. B: Cambio quístico definido por presencia de metaplasia escamosa del epitelio reducido del órgano del esmalte. Se observa una capa basal con células cilíndricas y cuboidales (flecha 1) que se hacen aplanadas a medida que avanza el estrato (flecha 2) (Tinción H-E 100x).

Se estandarizaron los diagnósticos determinándose que toda muestra que presente metaplasia escamosa del epitelio reducido del órgano del esmalte será considerado como cambio quístico temprano, esto según lo reportado en la literatura (Glosser \& Campbell, 1999; Adelsperger et al., 2000; Baykul et al., 2005; Saravana et al., 2008; Kotrashetti, 2010; Wali et al., 2012; Adaki et al., 2013). Se evaluó, además, la presencia de calcificaciones distróficas, restos epiteliales, infiltrado inflamatorio, densidad del conectivo y espesor del tejido.

Se realizó un análisis estadístico de prevalencia mediante proporción, intervalo de confianza de 95 $\%$, desviación estándar y análisis no paramétrico donde los resultados se analizaron con prueba de Chi cuadrado y test exacto de Fisher cuando había menos de 5 observaciones en una celda. Se consideraron resultados estadísticamente significativos aquellos que presenten un $p$ menor a 0,05. Para realizar el análisis de los datos se ocuparon programas computacionales como Microsoft Excel 2013 y STATA.

\section{RESULTADOS}

Sesenta y un muestras fueron analizadas, de las cuales $40(65 \%)$ correspondían a mujeres y 21 (35\%) a hombres. La distribución de edad de las muestras se encuentra en un rango entre los 12 y los 34 años con una media de edad de 20 años. Las mues- tras se distribuyeron en tres grupos etarios. El primero grupo etario estaba conformado por 37 muestras (61 $\%)$ de pacientes <20 años. El segundo grupo etario estaba conformado por 19 muestras (31\%) de pacientes entre 20 y 25 años. El tercer grupo etario estaba conformado por 5 muestras ( $8 \%$ ) de pacientes mayores a 25 años.

La distribución de las muestras según su ubicación correspondió a $27(44 \%)$ tejidos pericoronarios obtenidos del maxilar y 34 (56\%) tejidos pericoronarios obtenidos de la mandíbula.

Según el tipo de inclusión, las muestras correspondieron a 26 (43\%) tejidos pericoronarios obtenidos de terceros molares en inclusión mucosa y 35 (57 $\%$ en inclusión ósea. De acuerdo a la sintomatología local previa, 17 (28\%) pacientes que relataron haber tenido sintomatología local previa y 44 (72\%) no lo relataron al consultarles durante la anamnesis.

El diagnóstico histopatológico más prevalente fue Saco pericoronario infectado (SPI) con un $30 \%$ del total, seguido por aquellas muestras con presencia de "cambios quísticos tempranos (CQ)" con un 28 $\%$. El $26 \%$ de las muestras fueron diagnosticadas como Saco pericoronario fibrosado (SPF) y el $6 \%$ fueron diagnosticadas como Saco pericoronario normal (SPN). La Pericoronaritis crónica (PC) y la Pericoronaritis crónica fibrosada (PCF), en cambio, fueron los diagnósticos menos frecuentes con un $5 \%$ (Fig. 4).

La distribución de las variables histológicas arrojó presencia de calcificaciones distróficas en un $25 \%$ de las muestras, restos epiteliales odontogénicos en

\section{Diagnósticos}

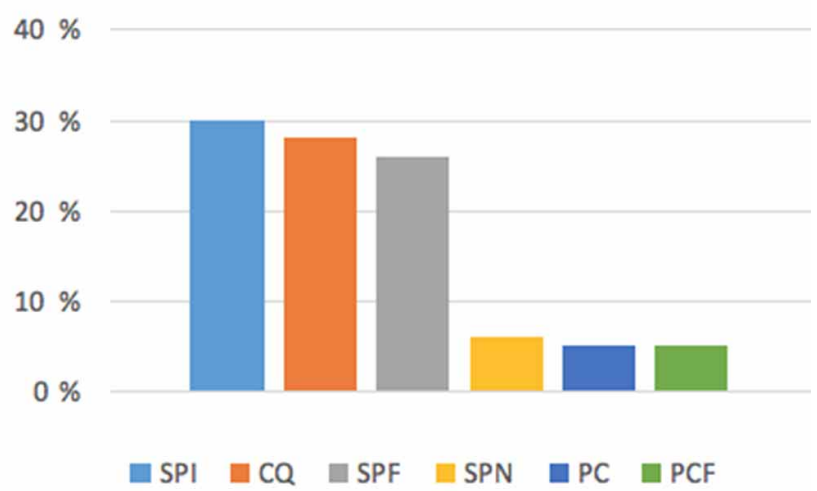

Fig. 4. Diagnósticos histopatológicos. 
el $84 \%$ e infiltrado inflamatorio crónico en un $62 \%$. En cuanto a la densidad tejido conectivo, el $89 \%$ presentaba un tejido conectivo denso y $11 \%$ tejido conectivo laxo. El espesor del tejido pericoronario se encontró aumentado solo en el $7 \%$ de las muestras. En relación al tipo de revestimiento epitelial, $72 \%$ de las muestras presentaban un epitelio reducido del órgano del esmalte (EROE) y $28 \%$ presentaban un epitelio escamoso estratificado (EEE), considerado como cambio quístico temprano (CQ). No se pesquisaron hallazgos histopatológicos sugerentes de patología tumoral.

La relación de los cambios quísticos tempranos (CQ) con las variables epidemiológicas demostró que la mayor frecuencia se encontró en pacientes menores de 20 años (53\%), género femenino (Fem) (53 $\%$ ), terceros molares en inclusión ósea (Inc.O) (59\%) y que no relataron sintomatología local previa (NoSLP) (59 \%). Sin embargo, no hubo significancia estadística en relación a estas variables. La única variable en alcanzar significancia estadística fue la ubicación, donde se encontró una frecuencia aumentada de cambios quísticos tempranos en terceros molares incluidos mandibulares (82\%).

\section{DISCUSIÓN}

Los terceros molares incluidos son el motivo de consulta de mayor demanda de los cirujanos bucales y máxilo-faciales. A pesar de que existe un consenso general en que los terceros molares incluidos deben ser extraídos cuando se observan cambios patológicos y síntomas clínicos, aún no existe consenso para la extracción profiláctica de terceros molares incluidos sanos.

Diferentes autores han demostrado la presencia de cambios quísticos tempranos en relación al capuchón pericoronario de terceros molares incluidos extraídos, sin sospecha de lesiones patológicas, clínica y radiográfica, lo que podría ser un factor a considerar como indicación quirúrgica de estos, donde los cambios microscópicos, evidenciados histológicamente, precederán a las alteraciones óseas características de una verdadera lesión quística.

En relación a los resultados obtenidos, la prevalencia de cambios quísticos tempranos fue de un $28 \%$. Este resultado es similar al encontrado por diversos autores como Glosser \& Campbell, quien en- contró un $32 \%$, Adelsperger et al., con $34 \%$ y Wali et al. y Adaki et al., quienes encontraron un $23 \%$ de los mismos. Incluso otros autores (Baykul et al.; Saravana et al.; Kotrashetti et al.) situaron cifras de $50 \%, 46 \%$ y $58 \%$ respectivamente.

Es de destacar que estudios realizados por AlKhateeb et al. (2006) y Güven et al. (2000), encontraron una amplia variedad de procesos neoplásicos como ameloblastomas, mixomas odontogénicos, fibromas odontogénicos, entre otros. Sin embargo, nuestro estudio no encontró lesiones compatibles con neoplasias.

La mayoría de los cambios quísticos tempranos (CQ) se encontraron en pacientes menores de 20 años (53 \%). A pesar de que este resultado no alcanzó significancia estadística, es similar al encontrado por Adelsperger et al., Kotrashetti et al. y Simsek-Kaya et al. (2011), quienes encontraron cambios quísticos con mayor frecuencia en pacientes menores a 21 años, entre 14-28 años y menores de 25 respectivamente. En cuanto al género, se encontró mayor frecuencia de cambios quísticos tempranos (CQ) en pacientes de género femenino (53\%), lo cual no alcanzó significancia estadística. Este resultado coincide con el encontrado por Glosser \& Campbell y por SimsekKaya et al. pero discrepa con la mayoría de los otros autores que han realizado estudios similares como Adelsperger et al., Baykul et al., Saravana et al., Kotrashetti et al., Wali et al. y Adaki et al., quienes encontraron mayor frecuencia de cambios quísticos en el género masculino.

En relación a la ubicación, la mayoría de los cambios quísticos se dieron en terceros molares incluidos mandibulares (82\%), siendo estadísticamente significativo. Este resultado coincide con el obtenido por Glosser \& Campbell, Güven et al. y Mesgarzadeh et al. (2008), sin embargo, discrepa con el obtenido por Adelsperger et al., quien encontró una frecuencia de cambios quísticos similares tanto para maxilar como para mandíbula.

Se observó una mayor cantidad de cambios quísticos tempranos en terceros molares con inclusión ósea (59\%), variable escasamente considerada en la literatura.

La mayor frecuencia de cambios quísticos tempranos (59\%), se encontraron en pacientes que no presentaron sintomatología local previa. No encontramos otros autores que vinculen esta variable, sin 
embargo, esperábamos encontrar que los cambios quísticos tempranos se correlacionasen con la presencia de sintomatología local previa. Esto permite asegurar que la ausencia de semiología en la zona no necesariamente demuestra estar libre de alteraciones, siendo una potencial indicación de extracción al menos en aproximadamente un tercio de los casos según nuestros resultados (28 \% cambios quísticos tempranos).

La característica de metaplasia escamosa del epitelio reducido del órgano del esmalte se puede presentar por diversos estímulos del conectivo, como por ejemplo la presencia de un infiltrado inflamatorio crónico o un tejido conectivo denso que genere algún grado de hipoxia del tejido pericoronario. Esta afirmación es respaldada por los resultados obtenidos en nuestro estudio, donde las muestras que presentaron metaplasia escamosa del epitelio reducido del órgano del esmalte, también presentaron infiltrado inflamatorio crónico y un tejido conectivo denso en altos porcentajes, estadísticamente significativos (82\% y 94 $\%$ respectivamente). Cabbar et al. (2008) explica que se ha informado de que la inflamación crónica puede causar irritación crónica estimulando la proliferación de células del epitelio oral, por lo cual, lo mismo podría suceder dentro del tejido pericoronario.

Es importante señalar que en la literatura existe alguna controversia en cuanto a lo que podríamos considerar como cambio quístico temprano del tejido pericoronario de un tercer molar incluido. Este estudio considero como factor determinante la presencia de metaplasia escamosa del epitelio reducido del órgano del esmalte, similar a lo expuesto por Glosser \& Campbell, Adelsperger et al., Baykul et al., Godoy et al. (2007), Saravana et al., Cabbar et al., Kotrashetti et al., Simsek-Kaya et al. y Wali et al.

Más aún, Adelsperger et al. y Cabbar et al. argumentan su afirmación mediante estudios de inmunohistoquímica a través de la expresión de marcadores celulares, cuya expresión en tejidos pericoronarios alcanzo un $62,5 \%$ para EEE (en contraste a $0 \%$ con EROE) lo que sugiere que la mayoría de los especímenes con metaplasia escamosa demostraron actividad proliferativa muy sugerente de transformación quística. Sobre lo mismo, Godoy et al. analizó inmunohistoquímicamente la expresión de ciertas integrinas en folículos dentales hiperplásicos y quistes dentígeros incipientes concluyendo que el primer evento de transformación quística es el cambio de metaplasia escamosa del epitelio reducido del esmal- te a epitelio estratificado. Sin embargo, existen autores como Daley \& Wysocki (1995), Edamatsu et al. (2005) y de Oliveira et al. (2011) que se oponen a estos planteamientos y consideran que la presencia de epitelio escamoso estratificado no debe ser diagnosticada ni sugerente de un cambio quístico sino que corresponde a un saco pericoronario con diferenciación escamosa propia del desarrollo y avance de la edad.

En resumen, diversas son las posibles indicaciones para realizar la exodoncia profiláctica de un tercer molar incluido clínicamente asintomático, donde un factor importante lo constituyen los posibles cambios patológicos que pueden ocurrir en los tejidos pericoronarios, los que si bien pueden tener una progresión incierta, podrían constituir un indicio más para indicar este tipo de extracciones.

\section{CONCLUSIONES}

El $28 \%$ de las muestras presentaron metaplasia escamosa del epitelio reducido del órgano del esmalte, considerada en la literatura como cambio quístico temprano.

Estos cambios histológicos son más frecuentes en pacientes menores de 20 años, género femenino, ubicación mandibular y en inclusión ósea.

La ausencia de síntomas asociados a un tercer molar incluido no significa necesariamente la ausencia de alguna variación histológica.

Existe relación estadísticamente significativa entre la presencia de infiltrado inflamatorio crónico, tejido pericoronario denso y la metaplasia escamosa.

PARRA, V. \& IBACETA, G. Histopathological findings of pericoronary tissue in healthy third molars. Int. J. Odontostomat., 13(3):332-337, 2019.

ABSTRACT: This study was performed to evaluate the presentation of early histopathological changes in pericoronal healthy third molars. Sixty-one pericororonitis tissues third molars, without radiographic evidence of abnormality in pericoronary sack, extracted for various reasons. The samples were fixed and processed routinely and stained with hematoxylin-eosin before evaluation. Diagnostic standardized concluding that all this shows that squamous metaplasia of the reduced enamel organ (EEE) is considered cystic epithelium were early change. Of the 
samples submitted, $28 \%$ showed squamous metaplasia early suggestive of cystic change. This histological tissue pericoronary variation was significantly higher in patients under 20 years of age, female gender, mandibular third molars, including in bone and in patients who reported no previous local symptoms $(P=0.05)$. These findings suggest that the absence of clinical and radiographic semiology third molars does not necessarily indicate the absence of alterations in tissue pericoronary thereof.

\section{histopathology.}

KEY WORDS: pericoronary tissue, third molar,

\section{REFERENCIAS BIBLIOGRÁFICAS}

Adaki, S. R.; Yashodadevi, B. K.; Sujatha, S.; Santana, N.; Rakesh, N. \& Adaki, R. Incidence of cystic changes in impacted lower third molar. Indian J. Dent. Res., 24(2):183-7, 2013.

Adelsperger, J.; Campbell, J. H.; Coates, D. B.; Summerlin, D. J. \& Tomich, C. E. Early soft tissue pathosis associated with impacted third molars without pericoronal radiolucency. Oral Surg. Oral Med. Oral Pathol. Oral Radiol. Endod., 89(4):4026, 2000.

Al-Khateeb, T. H. \& Bataineh, A. B. Pathology associated with impacted mandibular third molars in a group of Jordanians. $J$. Oral Maxillofac. Surg., 64(11):1598-602, 2006.

Baykul, T.; Saglam, A. A.; Aydin, U. \& Bas, ak, K. Incidence of cystic changes in radiographically normal impacted lower third molar follicles. Oral Surg. Oral Med. Oral Pathol. Oral Radiol. Endod., 99(5):542-5, 2005.

Cabbar, F.; Güler, N.; Comunoglu, N.; Sençift, K. \& Cöloglu, S. Determination of potential cellular proliferation in the odontogenic epithelia of the dental follicle of the asymptomatic impacted third molars. J. Oral Maxillofac. Surg., 66(10):2004-11, 2008.

Daley, T. D. \& Wysocki, G. P. The small dentigerous cyst. A diagnostic dilemma. Oral Surg. Oral Med. Oral Pathol. Oral Radiol. Endod., 79(1):77-81, 1995.

de Oliveira, M. G.; Lauxen Ida, S.; Chaves, A. C.; Rados, P. V. \& Sant'Ana Filho, M. Odontogenic epithelium: immunolabeling of Ki-67, EGFR and survivin in pericoronal follicles, dentigerous cysts and keratocystic odontogenic tumors. Head Neck Pathol., 5(1):1-7, 2011.

Divaris, K.; Fisher, E. L.; Shugars, D. A. \& White, R. P. Jr. Risk factors for third molar occlusal caries: a longitudinal clinical investigation. J. Oral Maxillofac. Surg., 70(8):1771-80, 2012.

Edamatsu, M.; Kumamoto, H.; Ooya, K. \& Echigo, S. Apoptosisrelated factors in the epithelial components of dental follicles and dentigerous cysts associated with impacted third molars of the mandible. Oral Surg. Oral Med. Oral Pathol. Oral Radiol. Endod., 99(1):17-23, 2005.

Glosser, J. W. \& Campbell, J. H. Pathologic change in soft tissues associated with radiographically 'normal' third molar impactions. Br. J. Oral Maxillofac. Surg., 37(4):259-60, 1999.

Godoy, G. P.; da Silveira, E. J.; Lins, R. D.; de Souza, L. B.; de Almeida Freitas, R. \& Queiroz, L. M. Immunohistochemical profile of integrins in enlarged dental follicles and dentigerous cysts. Oral Surg. Oral Med. Oral Pathol. Radiol. Endod., 104(6):e29-34, 2007.

Güven, O.; Keskin, A. \& Akal, U. K. The incidence of cysts and tumors around impacted third molars. Int. J. Oral Maxillofac. Surg., 29(2):131-5, 2000.
Kotrashetti, V. S.; Kale, A. D.; Bhalaerao, S. S. \& Hallikeremath, S. R. Histopathologic changes in soft tissue associated with radiographically normal impacted third molars. Indian J. Dent. Res., 21(3):385-90, 2010.

Mesgarzadeh, A. H.; Esmailzadeh, H.; Abdolrahimi, M. \& Shahamfar, M. Pathosis associated with radiographically normal follicular tissues in third molar impactions: a clinicopathological study. Indian J. Dent. Res., 19(3):208-12, 2008.

Moss, K. L.; Mauriello, S.; Ruvo, A. T.; Offenbacher, S.; White, R. P. Jr. \& Beck, J. D. Reliability of third molar probing measures and the systemic impact of third molar periodontal pathology. J. Oral Maxillofac. Surg., 64(4):652-8, 2006.

Phillips, C.; Norman, J.; Jaskolka, M.; Blakey, G. H.; Haug, R. H.; Offenbacher, S. \& White, R. P. Jr. Changes over time in position and periodontal probing status of retained third molars. J. Oral Maxillofac. Surg., 65(10):2011-7, 2007.

Saravana, G. H. \& Subhashraj, K. Cystic changes in dental follicle associated with radiographically normal impacted mandibular third molar. Br. J. Oral Maxillofac. Surg., 46(7):552-3, 2008.

Simsek-Kaya, G. Ozber E. Kalkan-Yaprer G. Dayr, E. Demirci, T. Soft tissue pathosis associated with asymptomatic impacted lower third molars. Med. Oral Patol. Oral Cir. Bucal, 16(7):e92936, 2011.

Wali, G. G.; Sridhar, V. \& Shyla, H. N. A study on dentigerous cystic changes with radiographically normal impacted mandibular third molars. J. Maxillofac. Oral Surg., 11(4):45865, 2012.

Direccion para correspondencia:

Dr. Victor Parra

Facultad Odontología UNAB

Cátedra Cirugía y Traumatología Máxilo-Facial

Santiago

CHILE

Email: vic.parra@hotmail.com

Recibido : 20-11-2018

Aceptado: 08-04-2019 